\title{
Article
}

\section{The Formation of Compact Elliptical Galaxies in The Vicinity of A Massive Galaxy: The Role of Ram-pressure Confinement}

Du, Min, Debattista, Victor P, Ho, Luis C., Cote, Patrick, Spengler, Chelsea, Erwin, Peter, Wadsley, James W., Norris, Mark, Earp, Samuel W.F. and Et, Al

Available at https://clok.uclan.ac.uk/28167/

Du, Min, Debattista, Victor P orcid iconORCID: 0000-0001-7902-0116, Ho, Luis C., Cote, Patrick, Spengler, Chelsea, Erwin, Peter, Wadsley, James W., Norris, Mark orcid iconORCID: 0000-0002-7001-805X, Earp, Samuel W.F. et al (2019) The Formation of Compact Elliptical Galaxies in The Vicinity of A Massive Galaxy: The Role of Ram-pressure Confinement. The Astrophysical Journal, 875 (1). p. 58. ISSN 0004-637X

It is advisable to refer to the publisher's version if you intend to cite from the work. http://dx.doi.org/10.3847/1538-4357/ab0e0c

For more information about UCLan's research in this area go to http://www.uclan.ac.uk/researchgroups/ and search for <name of research Group>.

For information about Research generally at UCLan please go to http://www.uclan.ac.uk/research/

All outputs in CLoK are protected by Intellectual Property Rights law, including Copyright law. Copyright, IPR and Moral Rights for the works on this site are retained by the individual authors and/or other copyright owners. Terms and conditions for use of this material are defined in the policies page. 


\title{
The Formation of Compact Elliptical Galaxies in the Vicinity of a Massive Galaxy: The Role of Ram-pressure Confinement
}

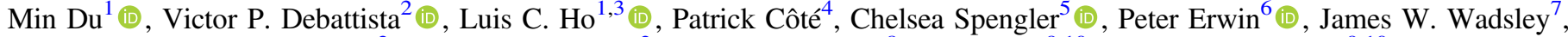 \\ Mark A. Norris ${ }^{2}$, Samuel W. F. Earp ${ }^{2}$, Thomas R. Quinn ${ }^{8}$, Karl Fiteni ${ }^{9,10}$, and Joseph Caruana ${ }^{9,10}$ \\ ${ }^{1}$ Kavli Institute for Astronomy and Astrophysics, Peking University, Beijing 100871, People's Republic of China; dumin@pku.edu.cn \\ 2 Jeremiah Horrocks Institute, University of Central Lancashire, Preston PR1 2HE, UK; vpdebattista@uclan.ac.uk \\ ${ }^{3}$ Department of Astronomy, School of Physics, Peking University, Beijing 100871, People's Republic of China \\ ${ }^{4}$ Herzberg Institute of Astrophysics, National Research Council of Canada, Victoria, BC V9E 2E7, Canada \\ ${ }_{5}^{5}$ Department of Physics and Astronomy, University of Victoria, Victoria, BC V8P 5C2, Canada \\ ${ }^{6}$ Max-Planck-Insitut für extraterrestrische Physik, Giessenbachstrasse, D-85748 Garching, Germany \\ ${ }^{7}$ Department of Physics \& Astronomy, McMaster University, Hamilton, L8S 4M1, Canada \\ ${ }_{9}^{8}$ Astronomy Department, University of Washington, Seattle, WA, USA \\ 9 Department of Physics, University of Malta, Msida MSD 2080, Malta \\ ${ }^{10}$ Institute of Space Sciences \& Astronomy, University of Malta, Msida MSD 2080, Malta \\ Received 2018 November 16; revised 2019 February 18; accepted 2019 March 6; published 2019 April 16
}

\begin{abstract}
Compact ellipticals (cEs) are outliers from the scaling relations of early-type galaxies, particularly the massmetallicity relation, which is an important outcome of feedback. The formation of such low-mass, but metal-rich and compact, objects is a long-standing puzzle. Using a pair of high-resolution $N$-body+gas simulations, we investigate the evolution of a gas-rich low-mass galaxy on a highly radial orbit around a massive host galaxy. As the infalling low-mass galaxy passes through the host's corona at supersonic speeds, its diffuse gas outskirts are stripped by ram pressure, as expected. However, the compactness increases rapidly because of bursty star formation in the gas tidally driven to the center. The metal-rich gas produced by supernovae and stellar winds is confined by the ram pressure from the surrounding environment, leading to subsequent generations of stars being more metal-rich. After the gas is depleted, tidal interactions enhance the metallicity further via the stripping of weakly bound, old, and metal-poor stars, while the size of the satellite is changed only modestly. The outcome is a metal-rich $\mathrm{cE}$ that is consistent with observations. These results argue that classical cEs are neither the stripped remnants of much more massive galaxies nor the merger remnants of normal dwarfs. We present observable predictions that can be used to test our model.
\end{abstract}

Key words: galaxies: dwarf - galaxies: evolution - galaxies: formation - galaxies: interactions - galaxies: ISM

\section{Introduction}

At the low-mass end of the early-type galaxy population, the well-known mass/luminosity-size relation (Larson 1981) splits into diffuse and compact branches. The compact branch is composed of compact ellipticals (cEs) and may even extend to the regime of ultra-compact dwarfs (Mieske et al. 2005; Haşegan et al. 2005; Chilingarian et al. 2007, 2009; Smith Castelli et al. 2008; Price et al. 2009; Huxor et al. 2011, 2013; Norris et al. 2014; Chilingarian \& Zolotukhin 2015; Guérou et al. 2015). cEs have effective (half-mass) radii $\left(R_{e}\right)$ that are generally less than $0.6 \mathrm{kpc}$, while their diffuse counterparts, the dwarf elliptical galaxies (dEs) or dwarf spheroidals (dSphs), have $R_{e} \sim 0.6-3 \mathrm{kpc}$ at similar masses. One $\mathrm{cE}$ formation scenario proposes that cEs are low-mass classical ellipticals, which is supported by the fact that they follow the same trend on the fundamental plane as the giant ellipticals (e.g., Wirth \& Gallagher 1984; Kormendy et al. 2009; Kormendy \& Bender 2012; Paudel et al. 2014). This implies formation through hierarchical mergers, as in "normal" ellipticals. Most cEs are notably more metal-rich than dEs (e.g., Chilingarian et al. 2009; Francis et al. 2012; Janz et al. 2016) and are outliers from the mass-metallicity relation of massive early-type galaxies (e.g., Gallazzi et al. 2005; Panter et al. 2008; Thomas et al. 2010) and low-mass galaxies in the Local Group (Kirby et al. 2013). Their dramatic offset from the mass-metallicity relation is an extraordinary challenge for the merger scenario.
An alternative formation scenario addresses the problem of high metallicity by proposing that cEs are the remnants of larger, more massive galaxies (e.g., Faber 1973; Bekki et al. 2001; Choi et al. 2002; Graham 2002). In this scenario, their disks are stripped via strong tidal interactions with an even more massive host galaxy, leaving only the compact, metal-rich bulges. For instance, the nearest $\mathrm{cE}$, and prototype of the class, M32, is known to be interacting with the Andromeda galaxy (M31); Choi et al. (2002) showed that M32's outer isophotes are distorted by M31's tidal field. However, the offset from the mass-metallicity relation of the dIrrs and dSphs is $\sim 0.4-0.6$ dex, (Gallazzi et al. 2005; Kirby et al. 2013), requiring a Milky Way-mass progenitor, whereas the total stellar halo of M31 is much less massive $\left(\sim 10^{9} M_{\odot}\right.$ level; e.g., Ibata et al. 2007, 2014; Courteau et al. 2011; Gilbert et al. 2012). Thus, no evidence for such a massive progenitor to M32 exists in M31. It is also worth noting that only the innermost regions (within $R_{e} / 8$ ) of massive, early-type galaxies show metallicities comparable to those of cEs (McDermid et al. 2015). Furthermore, by simulating the interaction between M31 and M32, Dierickx et al. (2014) showed that stripping from plausible progenitors was probably not sufficiently efficient. This is consistent with the kinematic measurement of M32 (Howley et al. 2013), which showed no evidence of tidal stripping within $1 \mathrm{kpc}$.

A dense cluster environment has usually been believed to play an essential role in generating cEs, because many of them 
are found in clusters. Recently, however, some cEs have been found in groups (Norris et al. 2014; Janz et al. 2016; FerréMateu et al. 2018) and even in the field (Huxor et al. 2013; Paudel et al. 2014; Chilingarian \& Zolotukhin 2015). The systematic study of compact low-mass galaxies in Norris et al. (2014) showed that cEs exist in a wide variety of environments. They suggested that the formation of cEs is likely to be associated with an adjacent massive host, with cEs in the field having been flung out of bound systems via three-body interactions (e.g., Chilingarian \& Zolotukhin 2015).

In this paper, we report on a numerical simulation specifically designed to explore the origin of $\mathrm{cE}$ galaxies around a large host via highly elliptical orbits. We simulate the evolution of a low-mass galaxy on a highly radial orbit around a massive disk galaxy. As a low-mass satellite falls into the hot corona of its host, the supply of metal-poor fresh gas is cut off, a process referred to as strangulation (Peng et al. 2010, 2015). A metal-poor dwarf might be expected to result. Here, we show that instead a low-mass satellite on such an orbit evolves into a metal-rich $\mathrm{cE}$ after several close flybys through the dense corona of its host.

The paper is organized as follows. Section 2 describes the simulation setup. In Section 3, we show the evolution of the morphology and kinematics of the low-mass satellite. Its rapid chemical enrichment due to ram-pressure confinement is presented in Section 4. In Section 5, we discuss the application of our work, the difference from previous simulations, and observational predictions. Our conclusions, observational predictions, and summary of our results are presented in Section 6.

\section{The Simulation}

We perform a simulation of a low-mass galaxy orbiting a massive galaxy. The low-mass galaxy starts at $200 \mathrm{kpc}$ from the larger galaxy with its velocity set for it to fall to within $\sim 20 \mathrm{kpc}$ of the host, giving it a highly eccentric orbit, which brings it inside the densest part of the hot gas corona. The motivation for a highly eccentric orbit is two-fold. Foremost, cEs are relatively rare objects, and therefore require unusual properties of some sort. Second, M32 is very close to M31, which requires its orbit to bring it close to the host.

Because we want to track the star formation (SF) and chemical enrichment of the galaxies, both galaxies start out as dark matter halos with gas but no star particles. The dark matter halos have Navarro-Frenk-White (Navarro et al. 1996) profiles, truncated exponentially beyond the virial radius. The dark matter halo is isotropic and is built using Eddington inversion (Kazantzidis et al. 2004). The dark matter halo of the massive host system has a virial radius of $r_{\mathrm{vir}}=198 \mathrm{kpc}$, concentration of $c_{\mathrm{vir}}=19$, and a virial mass of $M_{\text {vir }}=9.0 \times 10^{11} M_{\odot}$, while the low-mass system has $r_{\mathrm{vir}}=54 \mathrm{kpc}, \quad c_{\mathrm{vir}}=15$, and $M_{\mathrm{vir}}=1.8 \times 10^{10} M_{\odot}$. The halos of the host and low-mass galaxies are comprised of $5 \times 10^{6}$ and $10^{5}$ particles, respectively. The gas of the host galaxy has a similar profile with just $10 \%$ of the mass and is in thermal equilibrium. Because we do not wish to set the morphology of the dwarf by hand, we also include a corona for the dwarf galaxy; at this low mass, its corona cools rapidly and largely settles into a disk. The gas coronae have angular momenta such that the spin parameter $\lambda \approx 0.041$ for the massive host and $\lambda \approx 0.02$ for the low-mass system. Initially, there is an equal number of gas particles as dark particles. We use a spline softening of $\epsilon=103$ pc for dark matter particles and $\epsilon=50 \mathrm{pc}$ for gas and stars. The initial masses of gaseous and stellar particles are $1.8 \times 10^{5} M_{\odot}$ and $9.3 \times 10^{3} M_{\odot}$, respectively.

We do not introduce any stars into the initial conditions. Rather, all stars form self-consistently out of gas, ensuring that the chemical evolution is not imposed by the initial conditions. For the main galaxy, these initial conditions correspond to the state of the galaxy after the last major merger at redshift $z \sim 2$. Instead, for the dwarf galaxy, the corona quickly cools and settles into a disk. When the gas number density reaches 100 $\mathrm{cm}^{-3}$ and the temperature falls below $15,000 \mathrm{~K}$ in a converging flow, stars can form with a probability of $10 \%$ per dynamical time. A gas particle that loses more than $79 \%$ of its initial mass to SF will disperse its mass among its nearest neighbors and be removed. Each star particle represents an entire stellar population with a Miller-Scalo initial mass function (Miller \& Scalo 1979). Supernovae and stellar winds inject energy and metallicity into the interstellar medium (ISM) using the blastwave prescription of Stinson et al. (2006). Following Governato et al. (2010), $0.4 \times 10^{51} \mathrm{erg}$ is returned from each supernova. The gas starts with zero metallicity. Then, iron and $\alpha$-elements are produced in a subgrid model of Type Ia and II supernovae and asymptotic giant branch stars (Stinson et al. 2006) according to the yields of Raiteri et al. (1996), Thielemann et al. (1986), and Weidemann (1987). We include metal and thermal diffusion, based on a subgrid model of turbulence using the local smoothing length and velocity gradients (Smagorinsky 1963; Wadsley et al. 2008), with the diffusion parameter set to 0.03 .

The simulation is evolved for $10 \mathrm{Gyr}$ using GASOLINE2, a Smoothed Particle Hydrodynamics (SPH) code (Wadsley et al. 2004, 2017). This improved SPH code avoids the insufficient mixing of gas caused by contact discontinuities (see Wadsley et al. 2017). We use a base time step of $\Delta t=10 \mathrm{Myr}$ with time steps refined such that $\delta t=\Delta t / 2^{n}<\eta \sqrt{\epsilon / a_{g}}$, where $a_{g}$ is the acceleration at a particle's position and the refinement parameter $\eta=0.175$. We set the opening angle of the treecode gravity calculation to $\theta=0.7$. The time step of gas particles also satisfies the condition $\delta t_{\text {gas }}=\eta_{\text {courant }} h /\left[(1+\alpha) c+\beta \mu_{\text {max }}\right]$, where $\eta_{\text {courant }}=0.4, h$ is the SPH smoothing length set over the nearest 32 particles, $\alpha=1$ and $\beta=2$ are, respectively, the coefficients for the linear and quadratic terms of the artificial viscosity, and $\mu_{\max }$ is described in Wadsley et al. (2004). In order to help determine the effect of environment on the dwarf, we also evolve it in isolation.

\section{Evolution of the Satellite Galaxy}

Figure 1 (a) shows the trajectory of the satellite relative to the host galaxy, which forms an M31-like galaxy (e.g., Debattista et al. 2017); the evolution of the host will not be discussed here. The orbit decays rapidly due to dynamical friction, with its apocenter decreasing from 200 to $\sim 120 \mathrm{kpc}$ after the first pericentric passage and to $\sim 50 \mathrm{kpc}$ by the end of the simulation.

The low-mass system evolved in isolation loses its gaseous corona at a rate of $\sim 5 \times 10^{8} M_{\odot} \mathrm{Gyr}^{-1}(\sim 25 \%$ of the total gas mass per Gyr) in the first $2 \mathrm{Gyr}$, due to cooling, SF, and feedback. The loss of diffuse gas is even faster when the lowmass system is evolved in the orbit around the massive galaxy. 

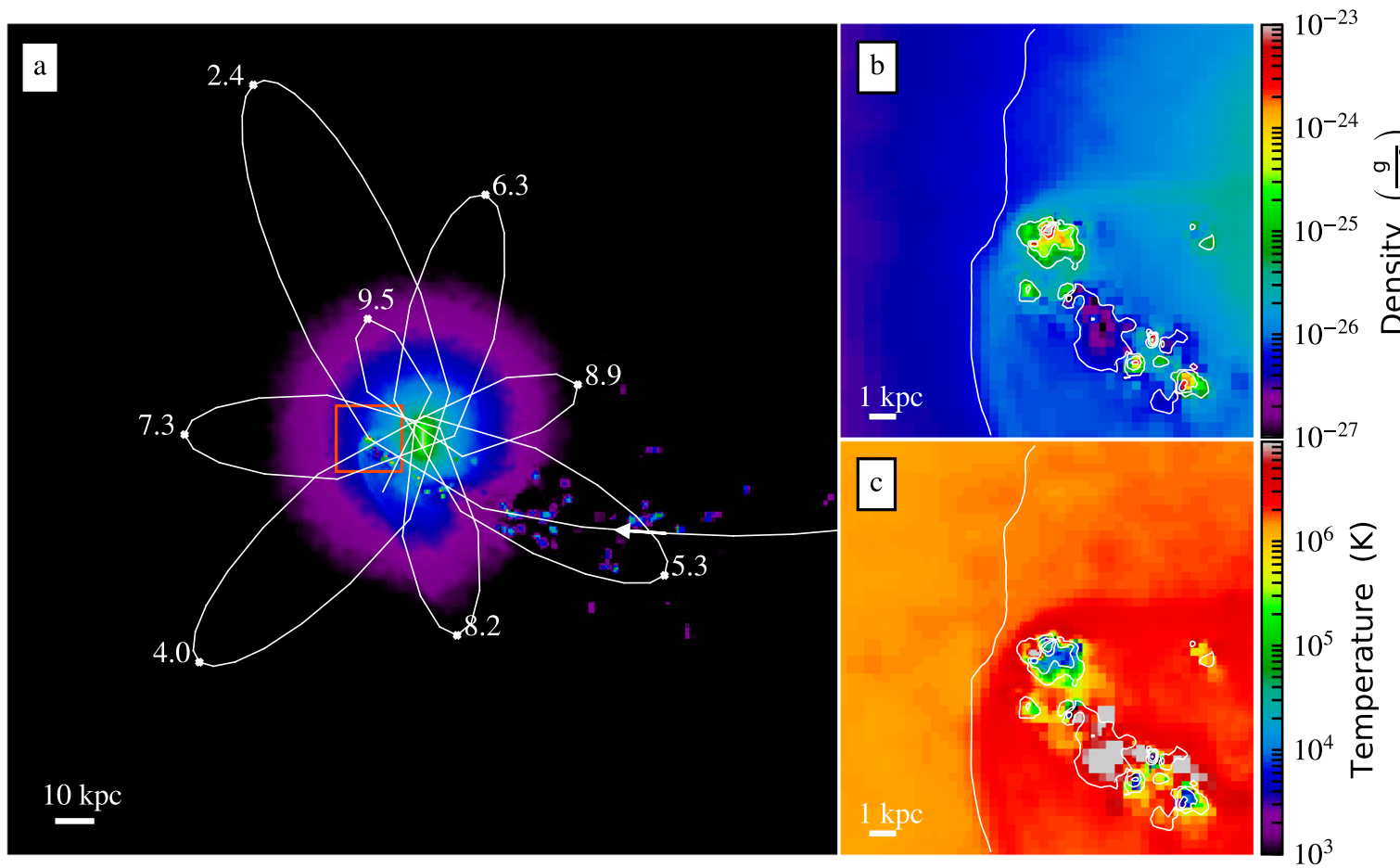

Figure 1. Panel (a) shows the trajectory of the low-mass satellite galaxy overlaid on a narrow slice of gas density at $t=1.66$ Gyr. The white dots mark the apocenters. The white arrow represents the position and motion of the satellite at $t=1.37 \mathrm{Gyr}$. At this point, both the host galaxy and the satellite galaxy are viewed nearly edgeon. While still more than $100 \mathrm{kpc}$ from the center of the host, the satellite has a stellar mass of $2.6 \times 10^{8} \mathrm{M}_{\odot}$ and has attained a velocity with respect to the host of $\approx 210 \mathrm{~km} \mathrm{~s}^{-1}$. Panels (b) and (c) are zoomed-in plots of the infalling satellite marked by the red square region in panel (a) at $t=1.66 \mathrm{Gyr}$. Panels (b) and (c) show the density and temperature of gas, respectively, overlaid with gas density contours. Panel (b) uses the same color bar as panel (a). At $t \sim 1.6 \mathrm{Gyr}$, the satellite first passes the pericenter at $\sim 16 \mathrm{kpc}$ from the center of the host. A bow shock is clearly visible in front of the satellite due to its supersonic bulk motion relative to the host corona. It never merges into the massive galaxy during the simulation, which lasts for $10 \mathrm{Gyr}$.

Before $t=1 \mathrm{Gyr}$, the satellite is still further than $150 \mathrm{kpc}$ from the center of the massive host, because it moves slowly (tens of $\mathrm{km} \mathrm{s}^{-1}$ level). Thus, the satellite evolves essentially by itself. Figure 2 reveals that, as a result, the satellite galaxy is very similar to the model evolved in isolation. The satellite's gaseous corona quickly cools and SF commences. Within a sphere of $5 \mathrm{kpc}$, about $\sim 60 \%$ of the baryonic mass is cold gas (temperature $T<15,000 \mathrm{~K}$ ) by $t=0.9 \mathrm{Gyr}$ and a disky dwarf of stellar mass $\sim 2 \times 10^{8} M_{\odot}$ forms (see Figure 2).

The satellite is affected as it approaches the massive galaxy via a number of mechanisms: (1) ram-pressure stripping of weakly bound gas (Gunn et al. 1972), (2) tidal stripping of stars, (3) triggering of SF within the remaining gas; (4) stellar heating via tidal stirring (Mayer et al. 2001). As the satellite sinks deeper into the host corona, it experiences an increased ram pressure and a very large fraction of gas in the diffuse outskirts is rapidly stripped by the ram pressure (e.g., Gunn et al. 1972). The satellite reaches the first pericenter at $\sim 1.6 \mathrm{Gyr}$, traveling at $\sim 400 \mathrm{~km} \mathrm{~s}^{-1}$; the sound speed in the host's corona is $\sim 160 \mathrm{~km} \mathrm{~s}^{-1}$, meaning the satellite's motion is highly supersonic. In panels (b) and (c) of Figure 1, we show magnified density and temperature images around the dwarf. A bow-shock structure (e.g., Domainko et al. 2006; Jachym et al. 2007) is distinguishable in front of the satellite due to its supersonic bulk motion relative to the corona. Nevertheless, the center retains a reservoir of cold gas.

This process repeats several times, with the orbit slowly decaying. By $t \approx 6.5 \mathrm{Gyr}$, after four pericentric passages, the satellite galaxy has lost all of its gas and is completely quenched. The satellite has a size typical of cEs for its mass, as shown in Figure 3, while the isolated model has a size similar
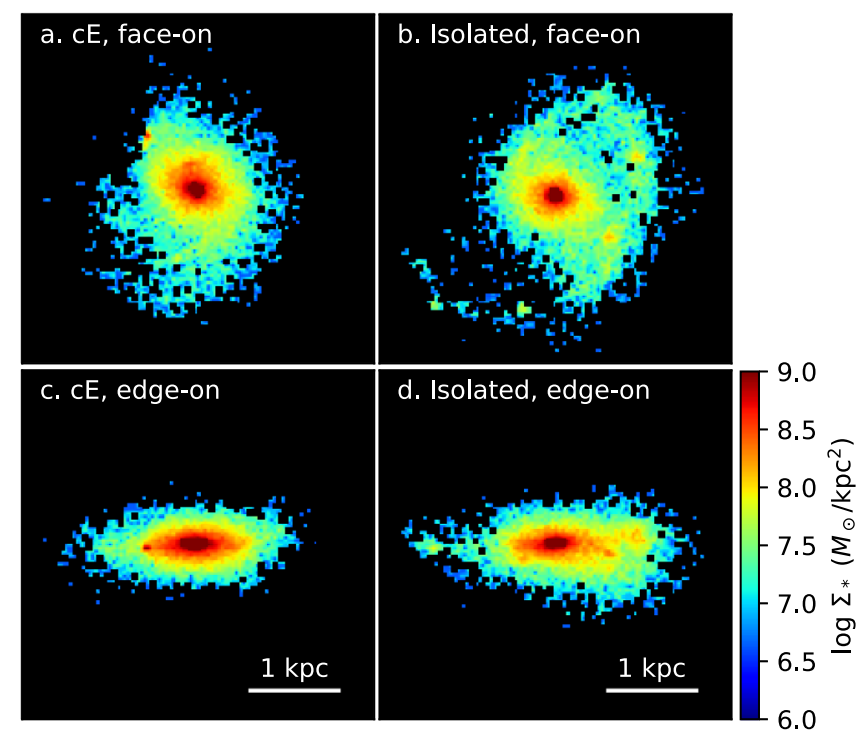

Figure 2. Stellar surface density distributions of the $\mathrm{cE}$ (left) and isolated (right) models at $t=0.9 \mathrm{Gyr}$ in the face-on (top) and edge-on (bottom) views. At this time, the $\mathrm{cE}$ has $M_{*} \simeq 2.0 \times 10^{8} M_{\odot}$ and $R_{e, *} \simeq 307 \mathrm{pc}$, while the isolated galaxy has $M_{*} \simeq 1.8 \times 10^{8} M_{\odot}$ and $R_{e, *} \simeq 446 \mathrm{kpc}$.

to dEs. At $t=10 \mathrm{Gyr}$, the satellite has reached a stellar mass of $M_{*} \simeq 2.7 \times 10^{8} M_{\odot}$ and a face-on effective radius of $R_{e, *}=144$ pc. In comparison, the isolated model forms a normal low-mass galaxy of $M_{*} \simeq 3.1 \times 10^{8} M_{\odot}$ and $R_{e, *} \simeq 1.5 \mathrm{kpc}$. The surface density distributions of the two models at $10 \mathrm{Gyr}$ are compared in Figure 4 . The isolated model still retains a cold gas reservoir of a mass of $3.5 \times 10^{8} M_{\odot}$ 


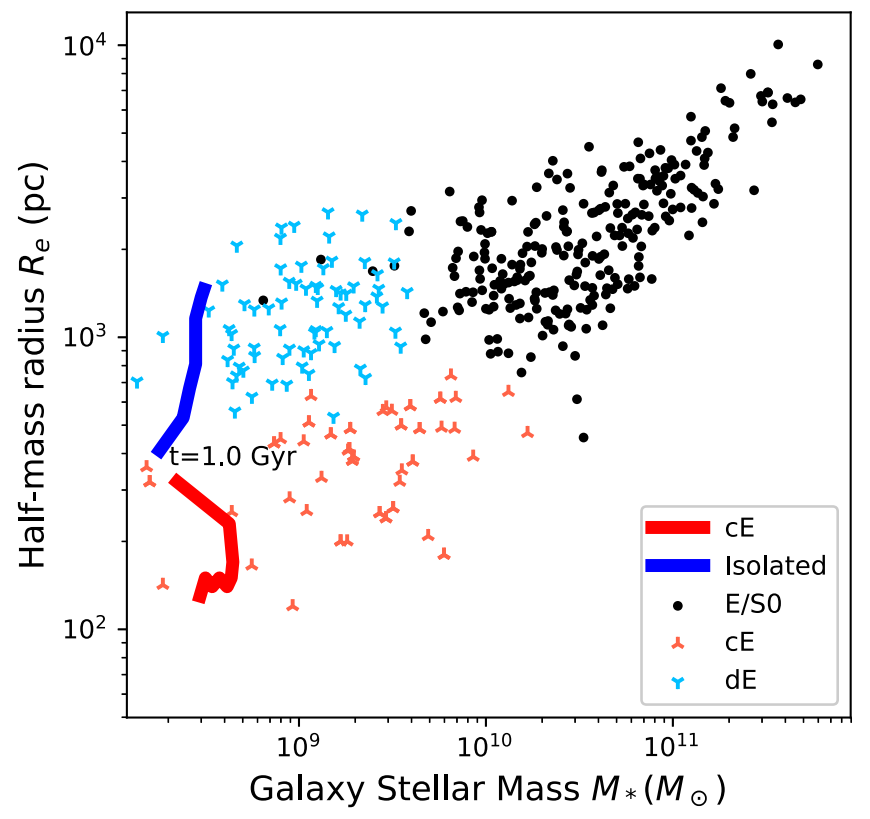

Figure 3. Evolution of the mass and size of the $\mathrm{cE}$ and isolated models overlaid on the observed mass-size relation. The data of elliptical/lenticular galaxies (E/S0s; black dots), cEs (red stars), and dEs (blue stars) are adopted from Janz et al. (2016). At $t=1 \mathrm{Gyr}$, the dwarf satellite has a mass and size similar to the isolated model. Its evolution in the vicinity of the massive host transforms it into a compact object.
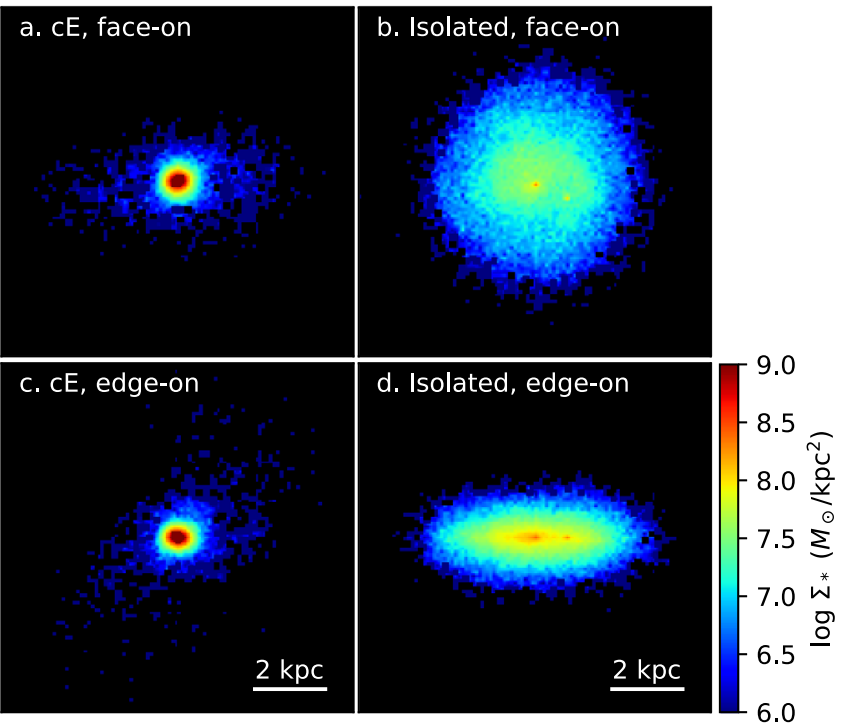

Figure 4. Stellar surface density distributions of the $\mathrm{cE}$ (left) and isolated (right) models at $t=10 \mathrm{Gyr}$ in the face-on (top) and edge-on (bottom) views. The satellite galaxy evolves into a $\mathrm{cE}$ after several close passages of the massive galaxy, while it becomes a normal diffuse galaxy in isolation. At this time, the $\mathrm{cE}$ has $M_{*} \simeq 2.7 \times 10^{8} M_{\odot}$ and $R_{e, *} \simeq 144 \mathrm{pc}$, while the isolated galaxy has $M_{*} \simeq 3.1 \times 10^{8} M_{\odot}$ and $R_{e, *} \simeq 1.5 \mathrm{kpc}$. Note the difference in their surface densities.

within $r<5 \mathrm{kpc}$. Thus, the isolated model is a typical diffuse and gas-rich dwarf galaxy.

\subsection{Morphology and Kinematics}

Figure 5 shows the evolution of some basic parameters of the satellite galaxy. The first panel presents the masses of stars, cold gas $\left(T<1.5 \times 10^{4} \mathrm{~K}\right)$, and dark matter. The second panel shows the distance between the satellite and the massive host.

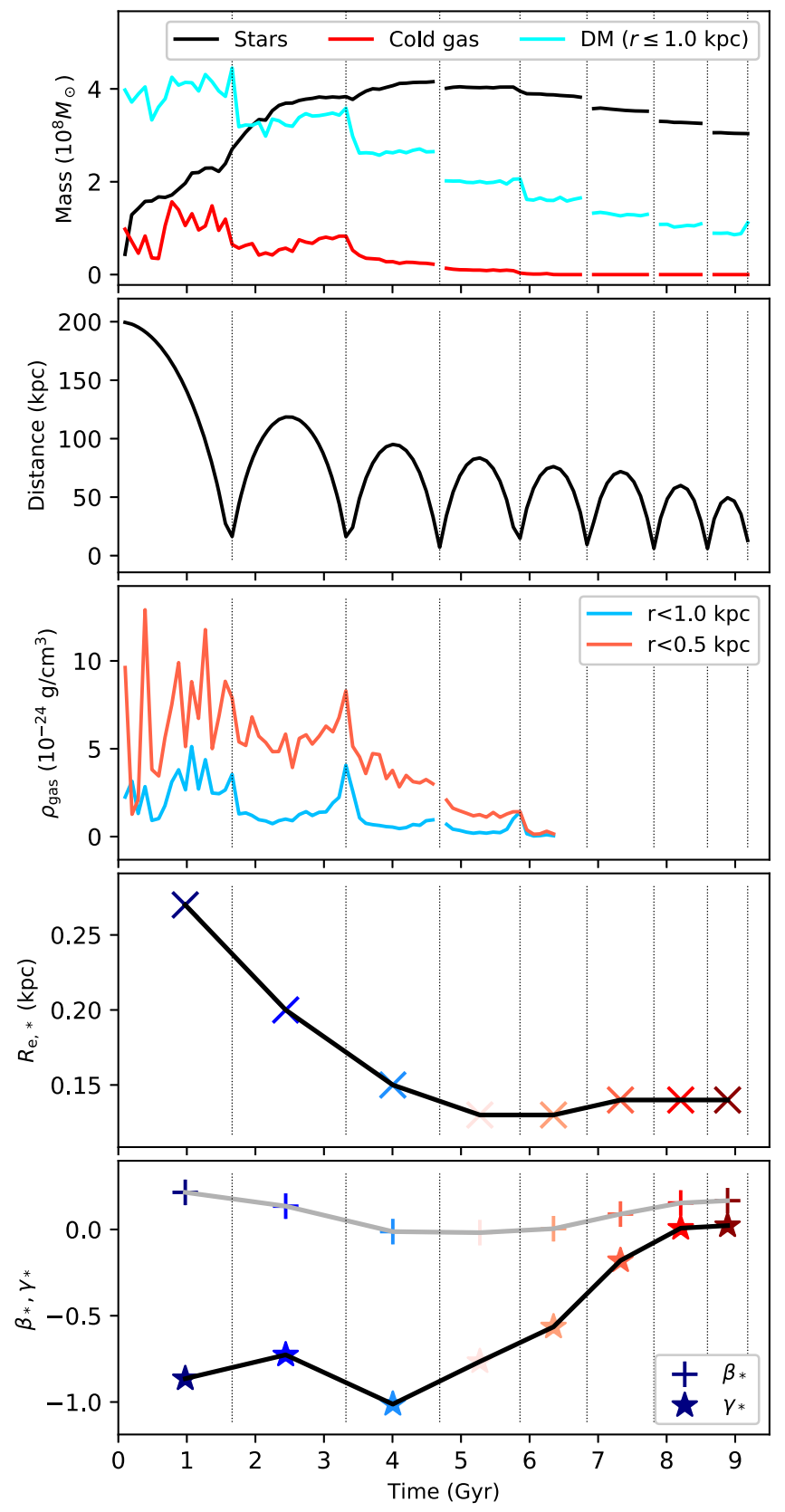

Figure 5. Evolution of the satellite galaxy. In the top panel, the black, red, and cyan lines represent the evolution of the total mass of stars, cold gas $(T<15,000 \mathrm{~K})$, and dark matter within $r<1.0 \mathrm{kpc}$, respectively. The second panel shows the separation between the satellite and the host galaxy. The vertical dotted lines mark the pericenters. The third panel shows the evolution of the cold gas density, $\rho_{\text {gas. }}$. The red and blue tracks represent the results obtained within a sphere of radius of 0.5 and $1.0 \mathrm{kpc}$, respectively. The data during passages at $<10 \mathrm{kpc}$ are excluded as they cannot be distinguished from the host galaxy. The fourth panel shows the stellar effective radius $\left(R_{e, *}\right)$ and the bottom panel the velocity dispersion anisotropies of the satellite measured face-on at apocenters. The blue and red markers in panels 4 and 5 represent stages (1) and (2), respectively. For comparison, we include the data point at $t=1.0 \mathrm{Gyr}$ when the satellite has not yet passed pericenter.

The vertical dotted lines in all of the panels mark the times of pericentric passages. The third panel of Figure 5 shows the mean density of cold gas, $\rho_{\text {gas }}$, within spheres of radii 0.5 and $1.0 \mathrm{kpc}$. The cold gas is compressed as the satellite moves close to its massive host, triggering starbursts. The fourth panel shows the effective radius, $R_{e, *}$. The fifth (bottom) panel shows the velocity dispersion anisotropies, $\beta_{*}$ and $\gamma_{*}$, of the satellite 


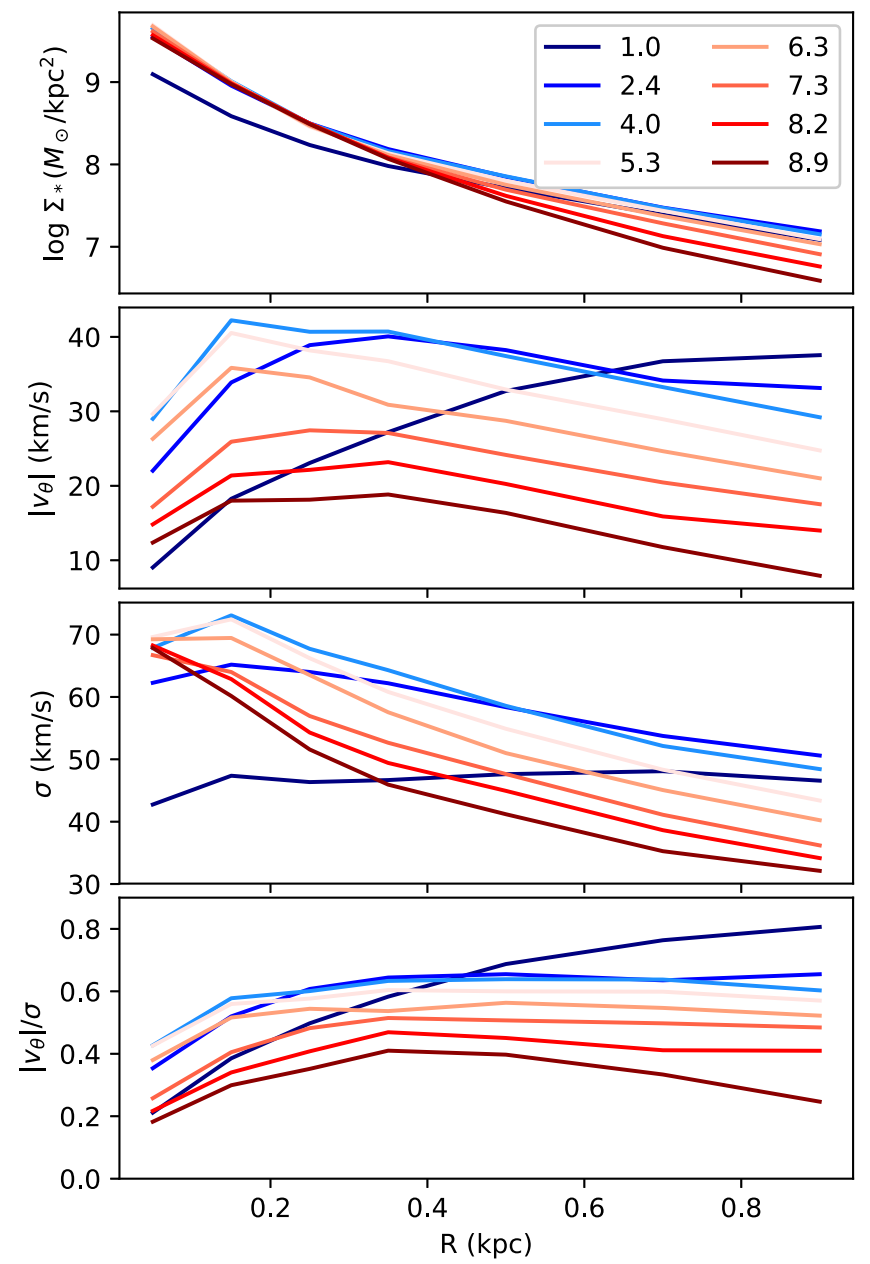

Figure 6. Radial profiles in cylindrical coordinates of the stellar surface density and kinematic properties of the satellite galaxy, measured at apocenters. From top to bottom, we show the surface density; the mean rotation speed, $\left|v_{\theta}\right|$; velocity dispersion, $\sigma=\sqrt{\sigma_{R}^{2}+\sigma_{\theta}^{2}+\sigma_{z}^{2}}$; and the ratio $\left|v_{\theta}\right| / \sigma$. The blue and red series of lines represent stages (1) and (2), respectively. The data point at $t=1.0$ Gyr shows the properties before the first pericentric passage.

measured at the apocenter. We define the anisotropies of the velocity dispersion ellipsoid as $\beta_{*}=1-\sigma_{\theta}^{2} / \sigma_{R}^{2}$ and $\gamma_{*}=1-\sigma_{z}^{2} / \sigma_{R}^{2}$, where both $\beta_{*}$ and $\gamma_{*}$ approach 0 as the system becomes isotropic. In Figure 6, we show the radial kinematics at the apocenters. Figures 5 and 6 reveal two distinct stages in the evolution of the satellite: (1) the compactness increases rapidly during 1-4 Gyr and (2) the mass and rotation decrease gradually during 4-10 Gyr. Stages (1) and (2) correspond to the blue and red series of lines, respectively, in Figure 6.

At $t=1 \mathrm{Gyr}$, the diffuse stellar distribution leads to a low $\sigma$ and a gently rising $\left|v_{\theta}\right|$ (Figure 6) with the radius. During stage (1), the compactness increases rapidly as a compact core is built up (the top panel of Figure 6) via rapid SF at the center. As suggested by previous simulations, both the high ram pressure (Bekki \& Couch 2003; Kronberger et al. 2008; Henderson \& Bekki 2016; Nehlig et al. 2016) and strong tidal interactions (e.g., Smith et al. 2010; Renaud et al. 2014; Nehlig et al. 2016) are likely to enhance the star formation rate (SFR; see also Section 3.2) by compressing the gas in the central region (see the third panel of Figure 5). At $t=4.5 \mathrm{Gyr}, M_{*}$ reaches its maximum value, $4.5 \times 10^{8} M_{\odot}$, while $R_{e, *}$ has dropped to $\approx 160$ pc. Such a compact concentration of mass results in a steeply rising rotation curve. The low-mass galaxy still retains a significant rotation at $t=4.0 \mathrm{Gyr}$, with $\left|v_{\theta}\right| / \sigma \sim 0.6$. Therefore, during stage (1), the infalling low-mass galaxy is transformed into a gas-poor and compact, but rapidly rotating, stellar system. Strong tidal interactions have only happened twice (at $t=1.7 \mathrm{Gyr}$ and at $3.3 \mathrm{Gyr}$ ) by this stage, but the stellar mass lost due to tidal stripping is not the most important factor driving the evolution of the dwarf up to this point, although dark matter is substantially stripped.

The growth of the stellar mass gradually declines because the cold gas is depleted, transitioning to stage (2). During this stage, $M_{*}$ decreases due to the loss of weakly bound stars via tidal stripping. However, tidal stripping has only a modest effect on the compactness, as shown by the small change in $R_{e, *}$ during 5-9 Gyr (the fourth panel of Figure 5). The remnant at $t=10 \mathrm{Gyr}$ has a similar mass, $M_{*} \simeq 2.7 \times 10^{8} M_{\odot}$, as the progenitor before its first pericentric passage. About $40 \%$ of the stellar mass is stripped with respect to the maximum $M_{*}$ at $t=4.5$ Gyr. Thus, tidal stripping of stars certainly plays a role, but it is not the dominant factor in the formation of this $\mathrm{cE}$. The satellite loses $\sim 75 \%$ of its dark matter due to the tidal interactions (the cyan profile in the top panel of Figure 5).

The kinematic properties and morphology of the infalling low-mass galaxy are also significantly changed during this stage. Strong tidal interactions heat stars impulsively via tidal stirring (e.g., Mayer et al. 2001), leading to a gradual decline in the rotation (Figure 6) and the anisotropies (the bottom panel of Figure 5). The satellite becomes a compact and kinematically isotropic stellar system dominated by random motions, with $\left|v_{\theta}\right| / \sigma \sim 0.3$, by $t=8.9 \mathrm{Gyr}$. We notice that M32 also has nearly isotropic orbital families, according to the result of the triaxial Schwarzschild modeling in van den Bosch \& de Zeeuw (2010).

When the low-mass galaxy evolves in isolation, both its effective radius and stellar mass increase continuously. It ends as a gas-rich, diffuse, fast-rotating $\left(\left|v_{\theta}\right| / \sigma \sim 0.8\right)$ galaxy that has a stellar mass comparable to that of the infalling low-mass galaxy.

\subsection{Bursty Star Formation}

In the cE model, the SFR is enhanced by compression of the ISM via tidal interactions and high ram pressure. The top panel of Figure 7 shows the SFR recovered from the stars left in the $\mathrm{cE}$ at $t=10 \mathrm{Gyr}$. The star formation history (SFH) of the isolated model is also shown for comparison. Due to the fact that the old stars in the cE are relatively weakly bound, they are more easily tidally stripped compared to the young stars; indeed $65 \%$ of stars formed before $t=1.3 \mathrm{Gyr}$ are stripped by 10 Gyr. This reduces the apparent SFR of stars older than 8.7 Gyr. The cE has a "bursty" SFH with multiple long-lasting starbursts separated by quiescent periods, which are consistent with its orbital period around the host. The bursty SF events are triggered at pericentric passages (vertical dotted lines), and last several hundreds of Myr, due to the combined effect of high ram pressure and tidally induced compression. Such a bursty SFH was also found in the satellite galaxies of the Illustris simulation (see Figure 7 of Mistani et al. 2016), but not in field galaxies. SF occurs randomly in the isolated model and rarely lasts a long time due to the modulating influence of feedback (Stinson et al. 2006). 

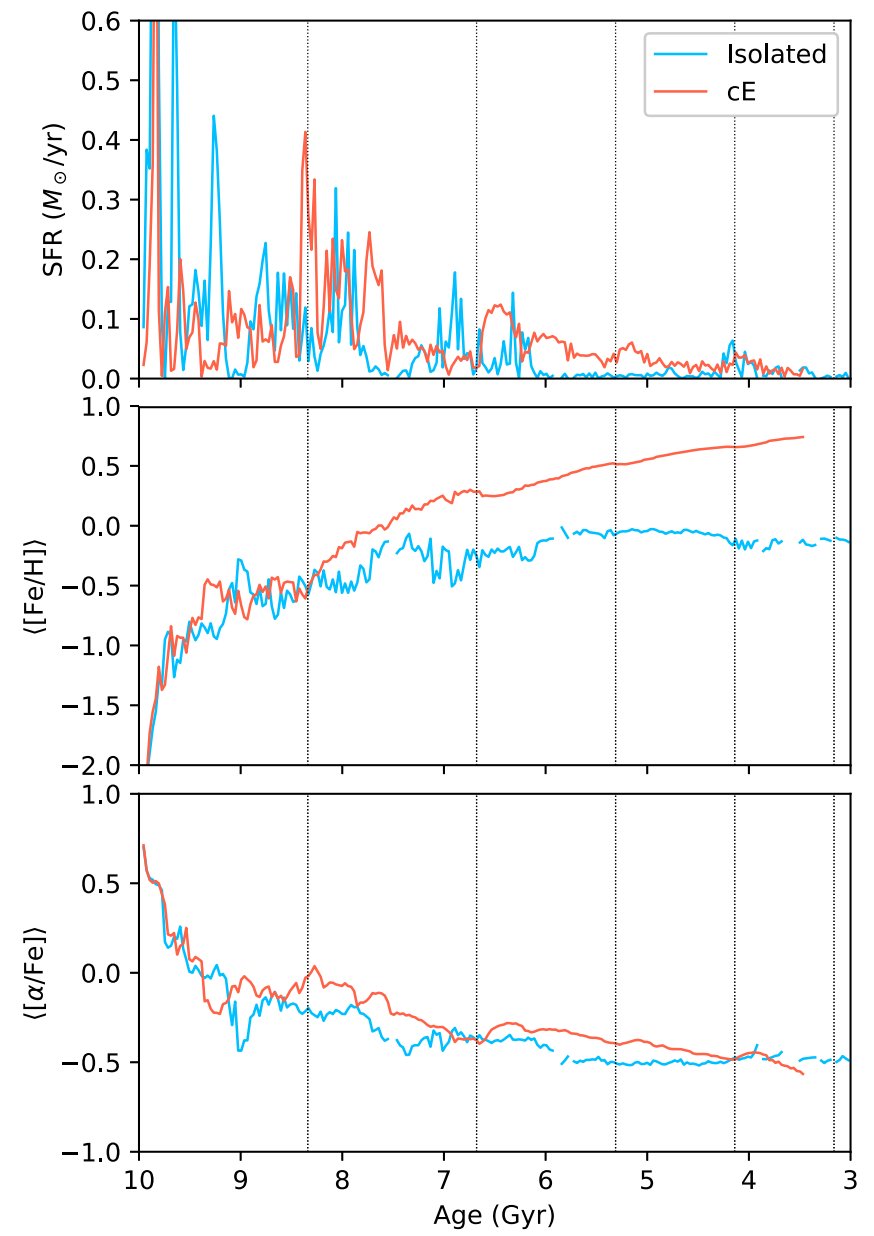

Figure 7. Evolution of the $\mathrm{cE}$ (red) and isolated models (blue) recovered from the final stellar population. From top to bottom, the panels show the SFR; the mass-weighted mean metallicity, $\langle[\mathrm{Fe} / \mathrm{H}]\rangle$; and $\alpha$-abundance, $\langle[\alpha / \mathrm{Fe}]\rangle$, as a function of age.

\section{Ram-pressure Confinement}

Generally speaking, cEs are significantly more metal-rich than galaxies of comparable masses. The origin of this high metallicity in cEs is crucial for understanding their formation. Metal-rich material, synthesized in stars, is returned via supernova feedback into the surrounding ISM. Some of this gas is retained, enriching the star-forming gas reservoir after cooling down. New stars then reflect the increased metallicity of the cool gas reservoir. The amount of outflowing gas that is lost depends on the ability of the galaxy to confine the hot gas created by supernova explosions. The shallow gravitational potential well of lower-mass galaxies results in metals being expelled more efficiently, producing the mass-metallicity relation (Carton et al. 2015). Janz et al. (2016) showed that cEs fall well above the relation between the escape velocity and metallicity for normal galaxies (Scott et al. 2009, 2013). Therefore, there must be an additional mechanism that allows cEs to retain their supernova-enriched hot gas. In this section, we investigate the confinement of metals in the cE model and compare it with that of the isolated model.

\subsection{Rapid Chemical Enrichment via Ram-pressure Confinement}

The middle and bottom panels of Figure 7 show the massweighted mean metallicity, $\langle[\mathrm{Fe} / \mathrm{H}]\rangle$, and $\alpha$-abundance, $\langle[\alpha / \mathrm{Fe}]\rangle$, of the stars at $t=10 \mathrm{Gyr}$ as a function of their age. Initially, $\langle[\mathrm{Fe} / \mathrm{H}]\rangle$ saturates at about -0.5 in both the isolated and satellite models, but after the first pericentric passage, the subsequent SF produces more metal-rich stars in the satellite, while the metallicity of stars in the isolated model rises only slightly. Just before quenching, the cE produces stars that are $\sim 1$ dex more metal-rich than in the isolated galaxy.

Figure 8 compares the chemical evolution of the two models in the $[\mathrm{Fe} / \mathrm{H}]-[\alpha / \mathrm{Fe}]$ space. The colors in panel (a) represent the formation times, $t_{\mathrm{f}}$, broadly binned by the times of pericentric passages at $1.56,3.32,4.69,5.76$, and $6.84 \mathrm{Gyr}$. Thus, each color corresponds to one bursty SF event triggered by a pericentric passage. The small gray dots correspond to stars with $t_{\mathrm{f}}<1.56 \mathrm{Gyr}$. The black contours show the distribution of the stars in the isolated galaxy at $t=10 \mathrm{Gyr}$. The oldest stars $\left(t_{\mathrm{f}}<1.56 \mathrm{Gyr}\right)$ in the $\mathrm{cE}$ (gray dots in panel (a) of Figure 8) follow a similar trend to those of the isolated galaxy (black contours), while the stars forming later follow a shallower trend (see also panels (b) and (c)). Both Figures 7 and 8 show that stars in the $\mathrm{cE}$ are noticeably more metal-rich than in the isolated model. Moreover, this is not just true on average, since the $\mathrm{cE}$ is able to form more metal-rich stars than in the isolated model.

Figure 8 also shows some unique features in the chemical evolution of the cE galaxy. Most prominently, the evolutionary track develops a break at the first pericenter, with the SF burst leading to $[\alpha / \mathrm{Fe}]$ rising rapidly. Subsequent SF occurs on an offset track. Several bursts of SF following the first pericentric passage lead to prominent "fingers," which do not alter the overall evolutionary trend until the next pericentric passage. At later pericentric passes, $\mathrm{SF}$ again leads to rapid rises in $[\alpha / \mathrm{Fe}]$, leading to further but weaker breaks.

The environment around the massive galaxy therefore plays an important role in not only triggering SF but also retaining the metals produced in supernovae. In Figures 9 and 10, we trace the gas particles heated by supernova explosions to follow the metal-rich outflows. We consider the gas particles that are heated to $>20,000 \mathrm{~K}$ from $<15,000 \mathrm{~K}$ within a short time $(<10$ Myr here $)$ when starbursts are on-going. Only the hot gas particles located within $0.5 \mathrm{kpc}$ of the center of the dwarf galaxy are used. Such gas particles are generally more metalrich than their surrounding environment as they obtain not only energy but also metals from supernovae. In the isolated model, we identify 71 such gas particles for a total mass $\simeq 1.6 \times 10^{6} M_{\odot}$. The longer lasting starburst in the $\mathrm{cE}$ heats more gas to high temperatures. As a consequence, 235 heated gas particles (mass $\left.\simeq 5.2 \times 10^{6} M_{\odot}\right)$ are identified.

In Figure 9, we mark the projected position of such gas particles (black dots). The white crosses represent the positions of the heated gas particles $0.5 \mathrm{Gyr}$ later. We define the radius containing $50 \%$ of these particles $0.5 \mathrm{Gyr}$ later as $r_{50}$, corresponding to the dashed circles in Figure 9. Similarly, $r_{80}$ (dotted circles) represents the radius containing $80 \%$ of the heated particles. $r_{50}$ and $r_{80}$ of the selected gas particles in the $\mathrm{cE}$ are 0.4 and $1.5 \mathrm{kpc}$, respectively, which is substantially smaller than in the isolated model $(2.6$ and $22.2 \mathrm{kpc})$. As a result, the metal-rich gas is less efficiently expelled by feedback in the $\mathrm{cE}$ model. In the $\mathrm{cE}$, the outskirts of the gas distribution 

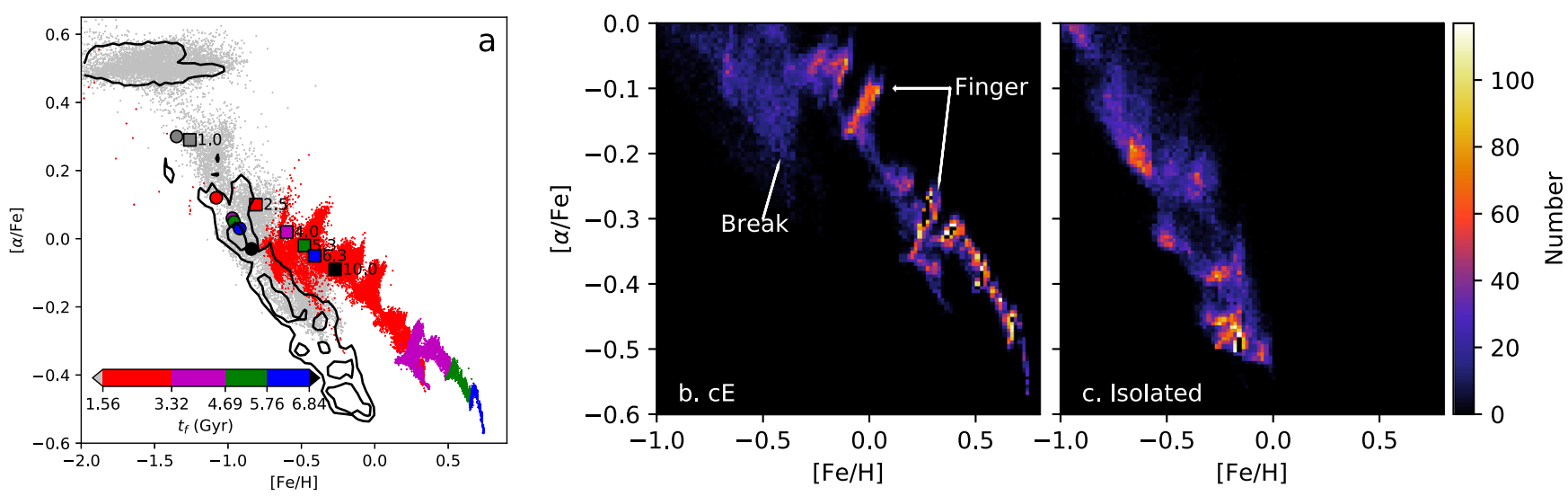

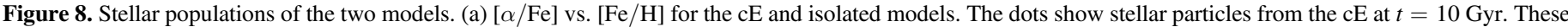

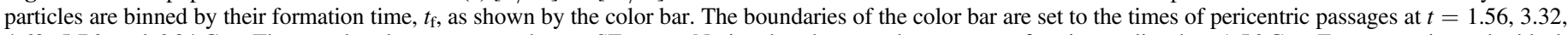

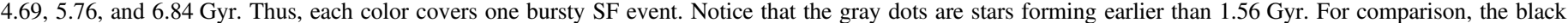

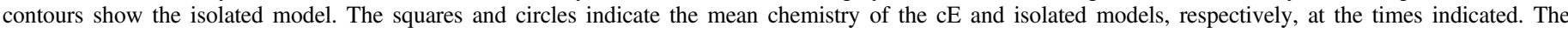

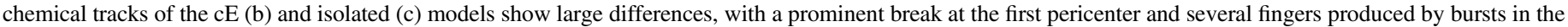
$\mathrm{cE}$ and a less discontinuous evolution in the isolated model.

is stripped backward by ram pressure (Figure 9(a)). In comparison, metal-rich outflows are present perpendicular to the disk (Figure 9(b)) in the isolated model.

Figure 10 follows the evolution of the radial distribution of the selected gas particles. We classify them into "outflow" (red), "fountain" (gray), and "remainer" (blue) groups. The gas of the outflow group escapes to $r>5 \mathrm{kpc}$, while the members of the remainer group never move beyond $1 \mathrm{kpc}$ from the center of the dwarf. The rest of the gas particles, except for depleted ones, belong to the fountain group. Their mean radii are shown by dashed lines, and the shaded regions correspond to their $1 \sigma$ envelopes. In the $\mathrm{cE}$, a majority of the gas particles are confined to the central region, where they can form new stars. About $35 \%$ of the selected gas particles are completely depleted by SF during this period. Furthermore, many fountain gas particles fall back to the center. Only $\sim 6 \%$ of the selected gas particles are lost, in the direction opposite to the cE's bulk motion, where the ram pressure is low. The majority of the gas is confined to a relatively small volume by the ram pressure, allowing rapid enrichment in the $\mathrm{cE}$. In contrast, most of the hot gas particles in the isolated model escape to $>1 \mathrm{kpc}$. As a consequence, the stellar metallicity of the isolated model increases much more slowly.

Figure 11 compares the stellar mass and metallicity of the models to observations. The cE's metallicity increases rapidly during stage (1) $(t<4.0 \mathrm{Gyr})$. Considering that the measured metallicity is affected by the region observed, we show the metallicity within 0.5 and 2 effective radii. The metallicity measured at the central region is higher because of the steep radial gradient. The galaxy evolved in isolation is significantly less metal-rich.

It is clear that the high ram pressure plays a crucial role in confining the metals released in supernova events. Under high ram pressure, metals are synthesized and recycled highly efficiently. This allows new stars to reach a high metallicity by confining the metal-rich gas in close proximity to the $\mathrm{cE}$.

\subsection{The Effect of Tidal Stripping}

Due to the fact that the old stars (those formed before the first pericentric passage) in the $\mathrm{cE}$ are relatively weakly bound, they are easily stripped with respect to the young stars forming in the central region. Since old stars are more metal-poor and $\alpha$ enhanced, this loss enhances the average metallicity further, while reducing $[\alpha / \mathrm{Fe}]$. $65 \%$ of stars forming before $t=1.3 \mathrm{Gyr}$ are stripped by $10 \mathrm{Gyr}$. As a consequence, the overall metallicity of the cE keeps increasing during stage (2) from 4 to $10 \mathrm{Gyr}$, as seen in Figure 11. In comparison, the metallicity in the isolated model changes little after 4 Gyr. Tidal stripping reduces the $\alpha$-abundance faster in the $\mathrm{cE}$ than in the isolated model (Figure 8), although the stars forming via the bursty SF in the $\mathrm{cE}$ are more $\alpha$-abundant (see the third panel of Figure 7).

\section{Discussion}

The scenario presented here relies on cEs forming in close proximity to a larger halo. This scenario is likely to be even more efficient in cluster environments where a low-mass satellite can reach a higher velocity, and the confining corona is hotter. The existence of cEs in the field (Huxor et al. 2013; Paudel et al. 2014; Chilingarian \& Zolotukhin 2015) is therefore likely a consequence of ejection in a three-body encounter between the $\mathrm{cE}$, the host galaxy, and a third galaxy (Chilingarian \& Zolotukhin 2015).

The broad metallicity distribution of low-mass galaxies suggests that the effect of environment is important. cEs are significantly offset from the normal mass-metallicity relation. We suggest that high ram pressure in high-speed passages around massive host galaxies confines metals in low-mass galaxies just when SF is enhanced because the gas is also compressed. To test the effect on the metallicity of the subgrid feedback, we tested the early part of the evolution using a stronger feedback model (super bubble feedback; Keller et al. 2014) and found that even in that case, the supernova ejecta are confined by ram pressure during the closest passage. Independently, Williamson \& Martel (2018) used wind tunnel experiments of dwarf galaxies to also demonstrate that ram pressure confines gas, and metals, to dwarf galaxies.

\subsection{Comparison with Previous Results}

Many studies have addressed the formation and evolution of low-mass dwarf satellites in the vicinity of a massive host (see the review Mayer 2010 and references therein). Ram pressure is 

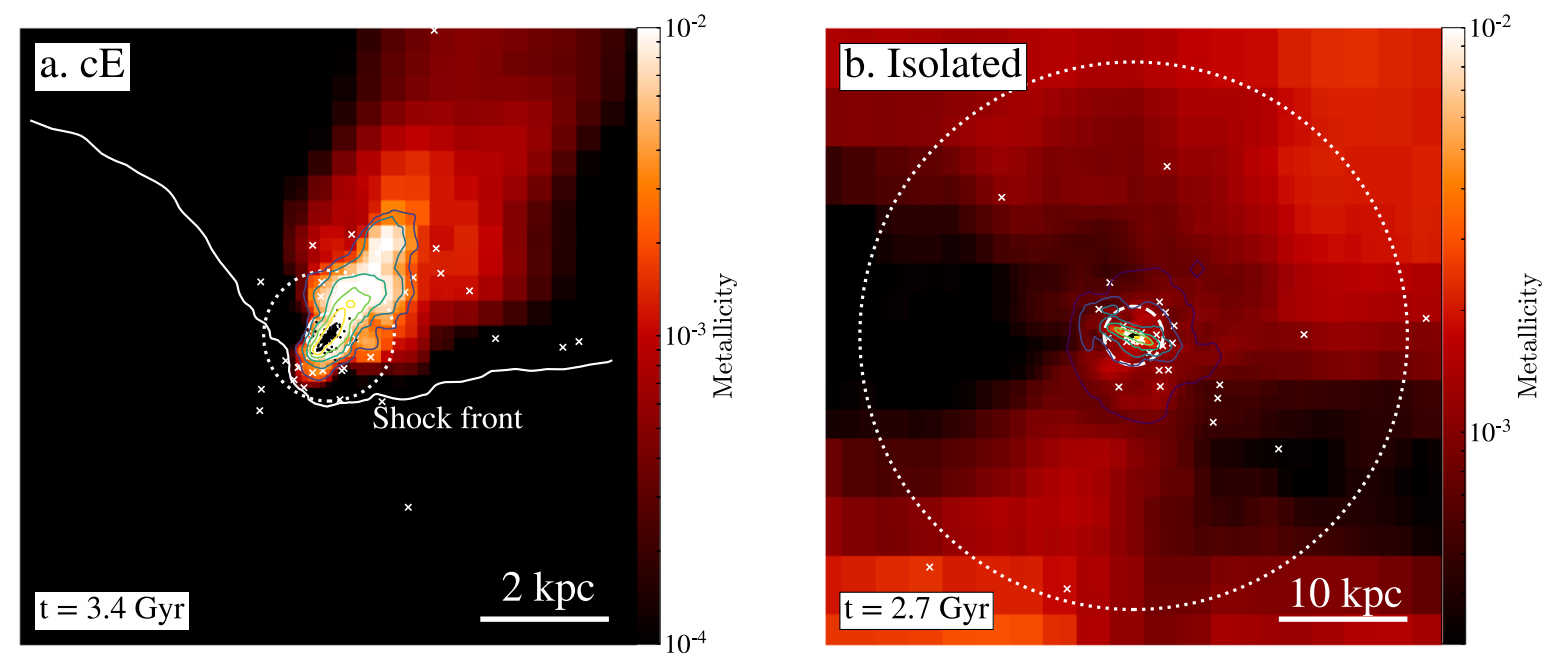

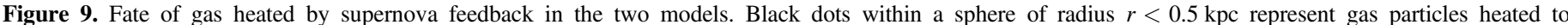

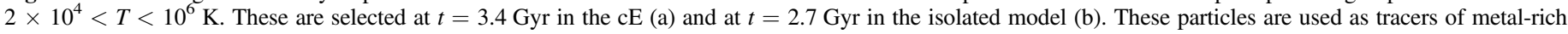

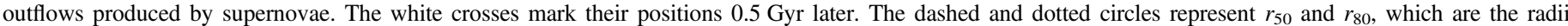

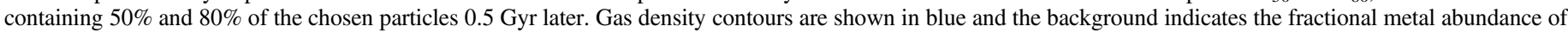
the gas. The thick white contour in panel a corresponds to the ram-pressure shock front. Note the different scale of the two panels.

often shown to strip gas dramatically in dwarf galaxies, then their disks are tidally heated to dSphs. In a cosmological context, Sawala et al. (2012) simulated the dwarf satellites in Milky Way-sized halos, finding a full family of dSphs/dEs formed. However, none of these studies have ever reported cEs produced by gas-rich dwarfs falling into the vicinity of a massive host. The absence of cEs is possibly because previous simulations have not, in general, used very eccentric orbits. Moreover, the resolution of cosmological simulations is possibly unsufficient in resolving cEs.

An exception was the work of Kazantzidis et al. (2017), who studied the evolution of disky dwarfs orbiting Milky Waysized hosts. They used pre-existing disks of varying gas fractions to represent the initial dwarfs; these had an initial scale length of $\approx 0.76 \mathrm{kpc}$, making them already extended dwarfs. In agreement with our results, they find that the initially gas-rich dwarf on a highly plunging orbit (their model S14) ends up slowly rotating, spherical, and gas-free. While they identify such galaxies as dSphs, Kazantzidis et al. (2017) do not discuss the compactness and metallicity of their dwarfs, and therefore it unclear whether they also form cEs under ram pressure confinement. However, an important difference in their models is that they already start with a quite extended disk.

In this paper, we present a possible scenario for the formation of metal-rich cEs from gas-rich, low-mass galaxies. In this scenario, a highly radial orbit of the satellite plays an important role in triggering a starburst and confining metals in the vicinity of a massive host. It is unclear what roles the mass and spin parameter $\lambda$ of the satellite play. Future work will explore a broader range of initial conditions. However, the differential comparison we performed between the isolated dwarf on the one hand and the satellite dwarf on the other, both of which have exactly identical initial conditions, suffices to highlight the physical influence of the environment.

Using a more massive and slowly spinning initial satellite will possibly favor the formation of cEs as well. In this work, we use $\lambda=0.02$. This is lower than the median value of $\sim 0.04$ (e.g., Bett et al. 2007), although $\lambda$ has a very large scatter in low-mass halos, varying from 0.01 to 1 . Moreover, Rodríguez-
Puebla et al. (2016) argue that $\lambda$ at high redshifts is lower by a factor of 2 than that at low redshifts. Thus, our choice of $\lambda$ is reasonable if the $\mathrm{cE}$ falls in at high redshifts. A systematic study is still required.

\subsection{Observational Consequences}

As shown in Figure 11, the cE model at $10 \mathrm{Gyr}$ exhibits comparable metallicity to observed cEs, while the isolated model is consistent with observed dEs. Furthermore, many cEs exhibit moderate, or even sub-solar, $[\alpha / \mathrm{Fe}]$ (the bottom panel of Figure 5 in Janz et al. 2016). In this scenario, it is reasonable that cEs exhibit very high metallicities but a wide range of $[\alpha /$ $\mathrm{Fe}$ ] due to the combined effect of ram-pressure confinement, tidal stripping, and bursty SF.

The trend of $[\alpha / \mathrm{Fe}]$ and $[\mathrm{Fe} / \mathrm{H}]$ can be used to constrain the formation of cEs. The break corresponds to the time that the $\mathrm{cE}$ first passes the pericenter, while the "fingers" represent bursts of SF after the pericenter. In between bursts, because the dwarf's gas is confined to a small volume, it is chemically well mixed, and stars form with a relatively small scatter. These features, being of the order of $\sim 0.1$ dex in $\alpha$-abundance, can be tested with future observations of M32.

\section{Summary}

We used a high-resolution simulation to investigate the evolution of a low-mass $\sim 10^{8} M_{\odot}$ galaxy infalling on a highly radial orbit around a massive disk galaxy. A compact, metalrich galaxy forms quickly due to the close flybys. We propose that a gas-rich, diffuse normal low-mass galaxy is replaced by a metal-rich $\mathrm{cE}$ within several Gyr. The evolution of the galaxy's structure and metallicity can be separated into two stages:

(1) While a large fraction of gas is stripped by ram pressure, the remaining gas in the center sustains bursty SF triggered by the combined effects of ram pressure confinement and tidal compression at pericentric passages. During periods of high ram pressure, the metal-rich outflows driven by supernovae and stellar winds are significantly suppressed. Thus, the infalling galaxy is able 

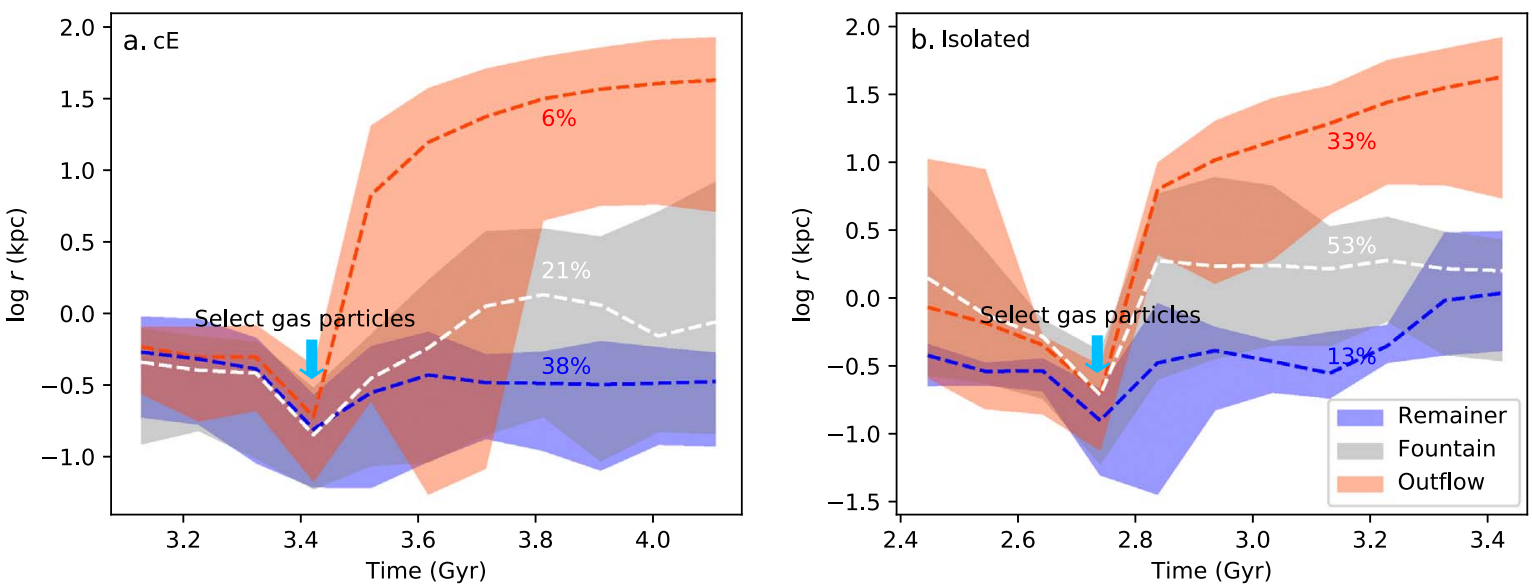

Figure 10. Evolution of the radial distribution of the hot gas particles heated by supernovae. We classify the particles into three groups according to their locations $0.5 \mathrm{Gyr}$ later. The gas particles escaping to $r>5 \mathrm{kpc}$ are classified as "outflow" (red), while the members of the "remainer" group (blue) are confined within $r<1 \mathrm{kpc}$. Except for particles depleted by SF, the rest of the gas particles belong to the "fountain" group. The dashed lines represent the mass-weighted mean radius in each group; the shaded regions correspond to the $1 \sigma$ envelopes. The mass fractions of each group are given in terms of the percentage. About $35 \%$ and $1 \%$ of the selected gas particles are depleted by $\mathrm{SF}$ in the $\mathrm{cE}$ and isolated models, respectively.

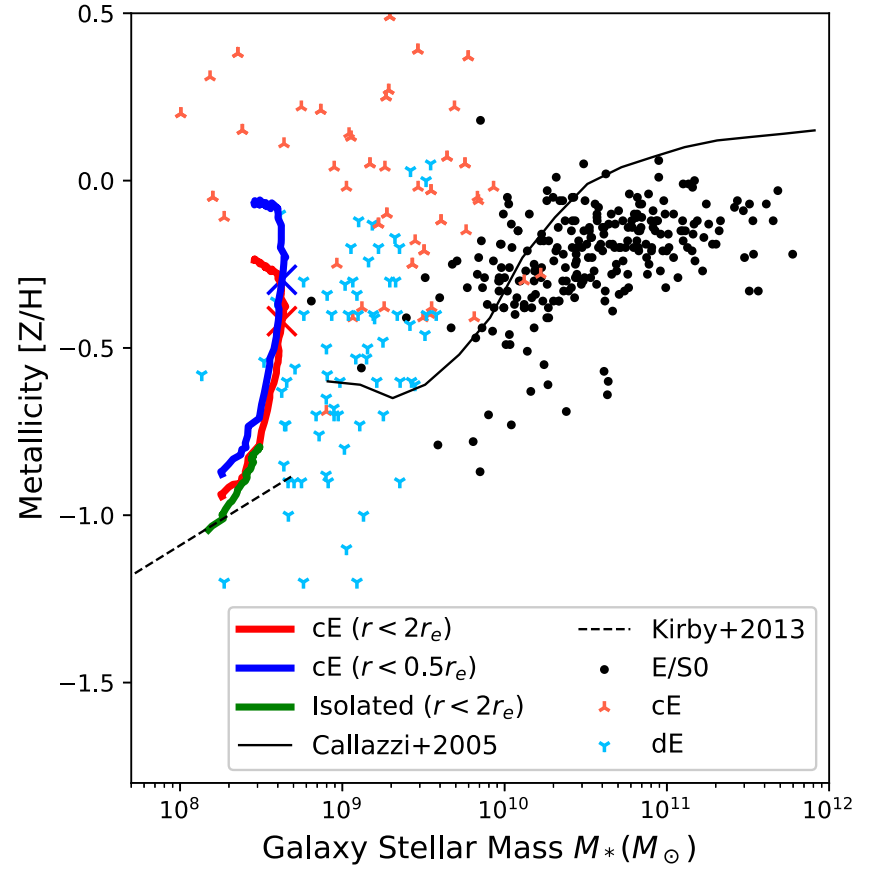

Figure 11. Evolution of the $\mathrm{cE}$ and isolated models overlaid on the observed mass-metallicity relation. The data of E/S0s (black dots), cEs (red stars), and dEs (blue stars) are adopted from Janz et al. (2016). The black solid and dashed lines correspond to the mass-metallicity relations of Gallazzi et al. (2005) and Kirby et al. (2013), respectively. The metallicity is measured in more central regions in Gallazzi et al. (2005), which leads to a higher metallicity than those of Janz et al. (2016). The red and blue tracks represent the chemical evolution of the $\mathrm{cE}$ model measured from stars within the 2 and 0.5 effective radii, respectively. The crosses mark $t=4 \mathrm{Gyr}$, which separates the two stages of the evolution of the $\mathrm{cE}$. The green track is the chemical evolution of the isolated model.

to retain more metals than in isolation, leading to the formation of a cE galaxy with high $[\mathrm{Fe} / \mathrm{H}]$.

(2) Following the quenching, frequent strong tidal interactions slowly change the mass, morphology, and metallicity of the $\mathrm{cE}$. The diffuse outskirts of the $\mathrm{cE}$, composed mainly of old metal-poor and $\alpha$-rich stars, are tidally stripped. The $\mathrm{cE}$ becomes less massive, more metal-rich, but less $\alpha$-enhanced due to tidal stripping. Tidal stirring gradually transforms the compact galaxy into a nearly isotropic object that is dominated by random motions.

The fact that cEs fall above the well-known mass-metallicity relation (e.g., Janz et al. 2016) has been considered as strong evidence that $\mathrm{cEs}$ are stripped remnants of massive galaxies. Indeed, cEs embedded in tidal streams have been considered as "smoking guns" of the tidal stripping scenario (Huxor et al. 2011). However, we have shown here that although tidal stripping plays a role in the formation of $\mathrm{cE}$ galaxies, it is not the most important mechanism. By tracing gas particles heated by supernovae, we verified that a high ram pressure environment confines more metals than when evolved in isolation. We suggest that the rapid metallicity enrichment in cEs is a natural outcome of the suppression of outflows and bursty SF for satellite galaxies orbiting in a dense corona around a massive host. The ram pressure confinement described here results in a very distinct and testable evolutionary track in $[\alpha / \mathrm{Fe}]-[\mathrm{Fe} / \mathrm{H}]$ space, with a shallower gradient following the cE's first pericentric passage; "finger-like" projections corresponding to SF bursts; and abrupt offsets to higher $[\alpha / \mathrm{Fe}]$ and $[\mathrm{Fe} / \mathrm{H}]$ at pericenters. This scenario implies that the progenitor does not need to be much more massive than the cE itself; as a result, the debris is likely to amount to a comparable mass as the satellite itself.

Ram-pressure confinement of metals likely affects galaxies in various types of dense environment, such as the vicinity of massive galaxies, rich groups, or clusters. In the past decades, ram pressure stripping has received appreciable attention. It is an efficient mechanism of stripping gas and suppressing SF, especially in the outer regions of satellite galaxies where gas is less bounded. Our results suggest that high ram pressure in the central regions of satellites sustains SF and leads to the buildup of a metal-rich core. This effect might be more important in low-mass satellites where the gravitational potential is otherwise not deep enough to maintain metals. It sheds new light on the evolution of satellite galaxies.

This work was supported by the National Key R\&D Program of China (2016YFA0400702) and the National Science Foundation of China $(11473002,11721303)$. The simulations in this paper were run at the DiRAC Shared Memory 
Processing system at the University of Cambridge, operated by the COSMOS Project at the Department of Applied Mathematics and Theoretical Physics on behalf of the STFC DiRAC HPC Facility (www.dirac.ac.uk). This equipment was funded by BIS National E-infrastructure capital grant ST/J005673/1, STFC capital grant ST/H008586/1, and STFC DiRAC Operations grant $\mathrm{ST} / \mathrm{K} 00333 \mathrm{X} / 1$. DiRAC is part of the National E-Infrastructure. The analysis was performed using yt (http://yt-project.org, Turk et al. 2011). This project is also supported by the High-performance Computing Platform of Peking University. M.D. is supported by the grants "National Postdoctoral Program for Innovative Talents" (No. $8201400810)$ and "Postdoctoral Science Foundation of China" (No. 8201400927) from the China Postdoctoral Science Foundation. V.P.D. was supported by STFC Consolidated grant ST/R000786/1. We also acknowledge support from a Newton Advanced Fellowship No. NA150272 awarded by the Royal Society and the Newton Fund.

Finally, I (M.D.) warmly thank my wife Pan Zhang for constant support and love, even when she was pregnant. Fan$\mathrm{Yu} \mathrm{Du}$, my son, hope you enjoy your journey to the universe, just like your name.

\section{ORCID iDs}

Min Du (i) https://orcid.org/0000-0001-9953-0359

Victor P. Debattista (1) https://orcid.org/0000-0001-7902-0116

Luis C. Ho iㅛ https://orcid.org/0000-0001-6947-5846

Chelsea Spengler (1) https://orcid.org/0000-0002-1685-4284

Peter Erwin (1) https://orcid.org/0000-0003-4588-9555

\section{References}

Bekki, K., \& Couch, W. J. 2003, ApJ, 596, 13

Bekki, K., Couch, W. J., Drinkwater, M. J., \& Gregg, M. D. 2001, ApJ, 557, L39

Bett, P., Eke, V., Frenk, C. S., et al. 2007, MNRAS, 376, 215

Carton, D., Brinchmann, J., Wang, J., et al. 2015, MNRAS, 451, 210

Chilingarian, I., Cayatte, V., Chemin, L., et al. 2007, A\&A, 466, L21

Chilingarian, I., Cayatte, V., Revaz, Y., et al. 2009, Sci, 326, 1379

Chilingarian, I., \& Zolotukhin, I. 2015, Sci, 348, 418

Choi, P. I., Guhathakurta, P., \& Johnston, K. V. 2002, AJ, 124, 310

Courteau, S., Widrow, L. M., McDonald, M., et al. 2011, ApJ, 739, 20

Debattista, V. P., Ness, M., Gonzalez, O. A., et al. 2017, MNRAS, 469, 1587

Dierickx, M., Blecha, L., \& Loeb, A. 2014, ApJL, 788, L38

Domainko, W., Mair, M., Kapferer, W., et al. 2006, A\&A, 452, 795

Faber, S. M. 1973, ApJ, 179, 423

Ferré-Mateu, A., Forbes, D. A., Romanowsky, A. J., Janz, J., \& Dixon, C. 2018, MNRAS, 473, 1819

Francis, K. J., Drinkwater, M. J., Chilingarian, I. V., Bolt, A. M., \& Firth, P. 2012, MNRAS, 425, 325

Gallazzi, A., Charlot, S., Brinchmann, J., White, S. D. M., \& Tremonti, C. A. 2005, MNRAS, 362, 41

Gilbert, K. M., Guhathakurta, P., Beaton, R. L., et al. 2012, ApJ, 760, 76

Governato, F., Brook, C., Mayer, L., et al. 2010, Natur, 463, 203

Graham, A. W. 2002, MNRAS, 334, 859

Guérou, A., Emsellem, E., McDermid, R. M., et al. 2015, ApJ, 804, 70
Gunn, J. E., Gott, J., \& Richard, I. 1972, ApJ, 176, 1

Haşegan, M., Jordán, A., Côté, P., et al. 2005, ApJ, 627, 203

Henderson, B., \& Bekki, K. 2016, ApJL, 822, L33

Howley, K. M., Guhathakurta, P., van der Marel, R., et al. 2013, ApJ, 765, 65

Huxor, A. P., Phillipps, S., \& Price, J. 2013, MNRAS, 430, 1956

Huxor, A. P., Phillipps, S., Price, J., \& Harniman, R. 2011, MNRAS, 414,3557

Ibata, R., Martin, N. F., Irwin, M., et al. 2007, ApJ, 671, 1591

Ibata, R. A., Ibata, N. G., Lewis, G. F., et al. 2014, ApJL, 784, L6

Jachym, P., Palous, J., Koppen, J., \& Combes, F. 2007, A\&A, 472, 18

Janz, J., Norris, M. A., Forbes, D. A., et al. 2016, MNRAS, 456, 617

Kazantzidis, S., Magorrian, J., \& Moore, B. 2004, ApJ, 601, 37

Kazantzidis, S., Mayer, L., Callegari, S., Dotti, M., \& Moustakas, L. A. 2017, ApJL, 836, L13

Keller, B. W., Wadsley, J., Benincasa, S. M., \& Couchman, H. M. P. 2014 MNRAS, 442, 3013

Kirby, E. N., Cohen, J. G., Guhathakurta, P., et al. 2013, ApJ, 779, 102

Kormendy, J., \& Bender, R. 2012, ApJS, 198, 2

Kormendy, J., Fisher, D. B., Cornell, M. E., \& Bender, R. 2009, ApJS, 182,216

Kronberger, T., Kapferer, W., Ferrari, C., Unterguggenberger, S., \& Schindler, S. 2008, A\&A, 481, 337

Larson, R. B. 1981, MNRAS, 194, 809

Mayer, L. 2010, AdAst, 2010, 278434

Mayer, L., Governato, F., Colpi, M., et al. 2001, ApJ, 559, 754

McDermid, R. M., Alatalo, K., Blitz, L., et al. 2015, MNRAS, 448, 3484

Mieske, S., Infante, L., Hilker, M., et al. 2005, A\&A, 430, L25

Miller, G. E., \& Scalo, J. M. 1979, ApJS, 41, 513

Mistani, P. A., Sales, L. V., Pillepich, A., et al. 2016, MNRAS, 455, 2323

Navarro, J. F., Frenk, C. S., \& White, S. D. M. 1996, ApJ, 462, 563

Nehlig, F., Vollmer, B., \& Braine, J. 2016, A\&A, 587, A108

Norris, M. A., Kannappan, S. J., Forbes, D. A., et al. 2014, MNRAS, 443, 1151

Panter, B., Jimenez, R., Heavens, A. F., \& Charlot, S. 2008, MNRAS, 391, 1117

Paudel, S., Lisker, T., Hansson, K. S. A., \& Huxor, A. P. 2014, MNRAS, 443, 446

Peng, Y., Maiolino, R., \& Cochrane, R. 2015, Natur, 521, 192

Peng, Y. J., Lilly, S. J., Kovač, K., et al. 2010, ApJ, 721, 193

Price, J., Phillipps, S., Huxor, A., et al. 2009, MNRAS, 397, 1816

Raiteri, C. M., Villata, M., \& Navarro, J. F. 1996, A\&A, 315, 105

Renaud, F., Bournaud, F., Kraljic, K., \& Duc, P.-A. 2014, MNRAS, 442, L33

Rodríguez-Puebla, A., Behroozi, P., Primack, J., et al. 2016, MNRAS, 462,893

Sawala, T., Scannapieco, C., \& White, S. 2012, MNRAS, 420, 1714

Scott, N., Cappellari, M., Davies, R. L., et al. 2009, MNRAS, 398, 1835

Scott, N., Cappellari, M., Davies, R. L., et al. 2013, MNRAS, 432, 1894

Smagorinsky, J. 1963, MWRv, 91, 99

Smith Castelli, A. V., Faifer, F. R., Richtler, T., \& Bassino, L. P. 2008, MNRAS, 391, 685

Smith, R., Davies, J. I., \& Nelson, A. H. 2010, MNRAS, 405, 1723

Stinson, G., Seth, A., Katz, N., et al. 2006, MNRAS, 373, 1074

Thielemann, F.-K., Nomoto, K., \& Yokoi, K. 1986, A\&A, 158, 17

Thomas, D., Maraston, C., Schawinski, K., Sarzi, M., \& Silk, J. 2010, MNRAS, 404, 1775

Turk, M. J., Smith, B. D., Oishi, J. S., et al. 2011, ApJS, 192, 9

van den Bosch, R. C. E., \& de Zeeuw, P. T. 2010, MNRAS, 401, 1770

Wadsley, J. W., Keller, B. W., \& Quinn, T. R. 2017, MNRAS, 471, 2357

Wadsley, J. W., Stadel, J., \& Quinn, T. 2004, NewA, 9, 137

Wadsley, J. W., Veeravalli, G., \& Couchman, H. M. P. 2008, MNRAS, 387,427

Weidemann, V. 1987, A\&A, 188, 74

Williamson, D., \& Martel, H. 2018, ApJ, 867, 72

Wirth, A., \& Gallagher, J. S. I. 1984, ApJ, 282, 85 